Cite this: Phys. Chem. Chem. Phys., 2013, 15, 262

Received 25th September 2012, Accepted 23rd October 2012

DOI: $10.1039 / \mathrm{c} 2 \mathrm{cp} 43381 \mathrm{~h}$

www.rsc.org/pccp

\section{Pyrazine excited states revisited using the extended multi-state complete active space second-order perturbation method $\dagger$}

\author{
Toru Shiozaki, $\neq^{* a}$ Clemens Woywod*b and Hans-Joachim Werner ${ }^{* a}$
}

\begin{abstract}
We demonstrate that the recently developed extended multi-state complete active space second-order perturbation theory (XMS-CASPT2) [Shiozaki et al., J. Chem. Phys., 2011, 135, 081106] provides qualitatively correct potential energy surfaces for low-lying excited singlet states of pyrazine, while the potential energy surfaces of the standard MS-CASPT2 methods are ill-behaved near the crossing point of two reference potential energy surfaces. The XMS-CASPT2 method is based on the extended multiconfiguration quasi-degenerate perturbation theory proposed earlier by Granovsky [J. Chem. Phys., 2011, 134, 214113]. We show that the conical intersection at the XMS-CASPT2 level can be described without artifacts if the entire method is invariant with respect to any unitary rotations of the reference functions. The photoabsorption spectra of the $1^{1} B_{3 u}$ and $1^{1} B_{2 u}$ states of pyrazine are simulated, based on a vibronic-coupling model Hamiltonian. The XMS-CASPT2 spectrum of the $1^{1} \mathrm{~B}_{3 \mathrm{u}}$ band is found to be comparable to the one computed by a more expensive multireference configuration interaction (MRCI) method, while the XMS-CASPT2 simulation of the $1^{1} \mathrm{~B}_{2 \mathrm{u}}$ band is slightly inferior to the $\mathrm{MRCl}$ one.
\end{abstract}

\section{Introduction}

There are many chemical processes that must be modeled by multireference electronic structure theories. Such processes include those related to state crossings and those dependent on global potential energy surfaces (PESs). Among others, the complete active space second-order perturbation (CASPT2) theories ${ }^{1-9}$ have been routinely used for such chemical applications. The CASPT2 method is based on a complete active space reference wavefunction and describes the so-called dynamical correlation using second-order perturbation theory. In CASPT2 the internal contraction scheme $\mathrm{e}^{10,11}$ is mostly used to reduce the dependence of the computational effort on the number of reference configurations, while some other variants of multireference perturbation theory use uncontracted configuration

\footnotetext{
${ }^{a}$ Institut für Theoretische Chemie, Universität Stuttgart, Pfaffenwaldring 55, D-70569 Stuttgart, Germany. E-mail: shiozaki@northwestern.edu, werner@theochem.uni-stuttgart.de; Fax: +49 711 68564442; Tel: +4971168564401

${ }^{b}$ Centre for Theoretical and Computational Chemistry, Department of Chemistry, University of Tromsø, N-9037 Tromsø, Norway.E-mail: woywod@ch.tum.de $\dagger$ Electronic supplementary information (ESI) available. See DOI: 10.1039/ c2cp $43381 \mathrm{~h}$

$\ddagger$ Present address: Department of Chemistry, Northwestern University, 2145 Sheridan Road, Evanston, IL 60208, USA.
}

state functions (CSFs) to expand the first-order wave functions. ${ }^{12-16}$ Recently, ${ }^{17}$ a CASPT2 method with density matrix renormalization group reference functions ${ }^{18,19}$ has also been reported. A concise review on the current status of CASPT2 can be found in ref. 20 .

One of the shortcomings of the CASPT2 method has been, however, that it is state-specific and it does not allow for a relaxation of the reference coefficients in the first-order wavefunction. This is problematic, especially near conical intersections and avoided crossings. In order to rectify this problem, a multi-state extension of the CASPT2 method (MS-CASPT2) has been developed by Finley et $a{ }^{5}{ }^{5}$ on the basis of the multiconfiguration quasi-degenerate perturbation theory (MCQDPT), ${ }^{15}$ which was in turn based on earlier developments. ${ }^{21}$ In MS-CASPT2, a second-order effective Hamiltonian is diagonalized at the end, which allows for mixings of the zeroth and first-order wave functions of different nearly degenerate states. There are also similar approaches called generalized Van-Vleck perturbation theories. ${ }^{22,23}$

Although MS-CASPT2 improves the description of electronic structures around conical intersections and avoided crossings in comparison to the single state CASPT2, there are still many cases where MS-CASPT2 PESs become singular at crossing points. $^{24}$ A remedy has been first proposed by Granovsky, ${ }^{25}$ who has proposed the 'extended' MCQDPT method, which is a generalization of the uncontracted MCQDPT of Nakano. ${ }^{15}$ 
An internally contracted CASPT2 variant has recently been implemented (XMS-CASPT2) by Shiozaki et al., together with its analytic nuclear energy gradients. ${ }^{26,27}$

The key idea in XMCQDPT and XMS-CASPT2 has been to use a zeroth-order Hamiltonian that is invariant with respect to any unitary rotations of the reference wavefunctions. For the second order energy, this is technically equivalent (see below) to use rotated reference functions, which diagonalize the Fock operator within the space spanned by the reference functions (or, the model space) when computing the perturbed wave functions. As a result, the computational cost of XMS-CASPT2 energy and nuclear gradient computations is the same as that of standard MS-CASPT2.

On the other hand, the XMS-CASPT2 method could introduce some shortcomings as well. For instance, the intruder state problems could be more pronounced, since in XMS-CASPT2 some states have a higher zeroth-order energy than in MS-CASPT2 due to diagonalization of the Fock operator. Furthermore, in rare cases when the reference functions span the complete active space, XMSCASPT2 transforms eigenfunctions of the active space Hamiltonian back to CSFs, which is not desirable. Therefore, it is important to carefully examine the accuracy of the XMS-CASPT2 method.

In order to assess the quality of PESs computed by the XMSCASPT2 approach we have in the current work employed this method to parametrize a vibronic coupling Hamiltonian for the $1^{1} B_{3 u}$ and $1^{1} B_{2 u}$ states of pyrazine. The strong vibronic interaction between these states is clearly manifested in the continuous wave $(\mathrm{CW})$ photoabsorption and resonance Raman spectra. The $1^{1} B_{3 u}-1^{1} B_{2 u}$ coupling has in fact become a showcase example for the calibration of vibronic coupling models. Spectroscopic properties of the two vibronically coupled electronic states have been studied in detail both by experiment ${ }^{28-32}$ as well as by theory. $^{33-49}$

We first present a summary of the XMS-CASPT2 theory (Section 2.1), followed by the methodology for the simulation of CW photoabsorption bands (Section 2.2). In Section 3.1 we show that the XMS-CASPT2 method provides smooth potential energy functions for the $1^{1} B_{3 u}$ and $1^{1} B_{2 u}$ states of pyrazine, which are free of artifacts and almost parallel to those computed by the multireference configuration-interaction approach, including the quasi-degenerate Davidson correction (MRCI+Q). In Section 3.2 it will be demonstrated that the invariant inclusion of level shifts ${ }^{50}$ in the XMS-CASPT2 theory is crucial for description of electronic structures near conical intersections. The simulations of the $1^{1} \mathrm{~B}_{3 \mathrm{u}}$ and $1^{1} \mathrm{~B}_{2 \mathrm{u}}$ photoabsorption bands performed with this model are discussed in Section 3.3 and compared with previous calculations, in particular ref. 48 and 49 . Conclusions based on the computational results are given in Section 4.

\section{Theoretical background}

In this section, we briefly review the XMS-CASPT2 theory and the framework of computing photoabsorption spectra. For details, the readers are referred to ref. 26 and 48, respectively.

\subsection{XMS-CASPT2 theory}

The main idea of the extended multistate multireference perturbation theories, first proposed by Granovsky, ${ }^{25}$ is to make the multistate theories invariant with respect to any unitary rotations of reference [i.e., complete-active-space self-consistentfield (CASSCF)] functions. As shown previously ${ }^{25,26}$ and herein, this is essential for a consistent simulation of conical intersections and avoided crossings.

The invariant form of the XMS-CASPT2 amplitude equations and the effective Hamiltonian is

$$
\begin{aligned}
& \left\langle\Omega\left|\hat{H}^{(0)}+E_{\text {shift }}\right| \Psi_{N}^{(1)}\right\rangle \\
& -\sum_{M}\left\langle\Omega \mid \Psi_{M}^{(1)}\right\rangle\left\langle M\left|\hat{H}^{(0)}\right| N\right\rangle+\langle\Omega|\hat{H}| N\rangle=0,
\end{aligned}
$$

$\left(H_{\mathrm{eff}}\right)_{M N}=\frac{1}{2}\left(\left\langle\Psi_{M}|\hat{H}| N\right\rangle+\left\langle\mathrm{M}|\hat{H}| \Psi_{N}\right\rangle\right)-E_{\text {shift }}\left\langle\Psi_{M}^{(1)} \mid \Psi_{N}^{(1)}\right\rangle$,

where $\Omega$ is the projection manifold, $\hat{H}$ is the electronic Hamiltonian, and $\left|\Psi_{N}\right\rangle \equiv|N\rangle+\left|\Psi_{N}^{(1)}\right\rangle$. The zeroth order Hamiltonian is defined as

$$
\hat{H}^{(0)}=\sum_{M N}|N\rangle\langle N|\hat{f}| M\rangle\langle M|+\hat{Q} \hat{f} \hat{Q} .
$$

$|M\rangle$ is the $M$ th state in an underlying CASSCF calculation, and $\hat{Q}$ is the projector $1-\sum_{M}|M\rangle\langle M|$. If internally contracted functions are used for $\left|\Psi_{N}^{(1)}\right\rangle$, the first-order wave function for each state should be expanded in terms of the union of the internally contracted functions generated from all the references ${ }^{26,51}$ (the so-called MS-MR-CASPT2 scheme in MOLPRO $\left.{ }^{52}\right) . \hat{f}$ is the generalized Fock operator

$$
\hat{f}=\sum_{r s}\left[h_{r s}+\sum_{i j} \gamma_{i j}^{a v}\left(J_{r s}^{i j}-\frac{1}{2} K_{r s}^{i j}\right)\right] \hat{E}_{r s}
$$

with the standard spin-free excitation operators $\hat{E}_{r s}$ and the state-averaged density matrix $\gamma_{i j}^{a v}$. Note that $i$ and $j$ label (doubly and partially) occupied orbitals, while $r$ and $s$ label any orbitals.

The level shift $E_{\text {shift }}$ has been introduced into CASPT2 in order to circumvent intruder state problems. ${ }^{50}$ We have introduced the level shift in the XMS-CASPT2 amplitude equations [eqn (1)] in an invariant way with respect to any unitary rotation of reference functions. The proof of invariance is straightforward. ${ }^{25}$ Thanks to this invariance, one can transform the reference functions by means of a unitary matrix $U_{M N}$ so that $|\tilde{N}\rangle=\sum_{M}|M\rangle U_{M N}$ diagonalizes $\hat{H}^{(0)}$, i.e.,

$$
\hat{H}^{(0)}=\sum_{N} \tilde{E}_{N}^{(0)}|\tilde{N}\rangle\langle\tilde{N}|+\hat{Q} \hat{f} \hat{Q}
$$

with

$$
\left\langle\tilde{M}\left|\hat{H}^{(0)}\right| \tilde{N}\right\rangle=\delta_{M N} \tilde{E}_{N}^{(0)} .
$$

The amplitude equations are then decoupled as

$$
\left\langle\Omega\left|\hat{H}^{(0)}-\tilde{E}_{N}^{(0)}+E_{\text {shift }}\right| \tilde{\Psi}_{N}^{(1)}\right\rangle+\langle\Omega|\hat{H}| \tilde{N}\rangle=0,
$$

and the effective Hamiltonian becomes

$$
\left(H_{\mathrm{eff}}\right)_{M N}=\frac{1}{2}\left(\left\langle\tilde{\Psi}_{M}|\hat{H}| \tilde{N}\right\rangle+\left\langle\tilde{M}|\hat{H}| \tilde{\Psi}_{N}\right\rangle\right)-E_{\text {shift }}\left\langle\tilde{\Psi}_{M}^{(1)} \mid \tilde{\Psi}_{N}^{(1)}\right\rangle,
$$


where $\left|\tilde{\Psi}_{N}^{(1)}\right\rangle=\sum_{N}\left|\Psi_{N}^{(1)}\right\rangle U_{N M}$ and $\tilde{\Psi}_{N}=|\tilde{N}\rangle+\left|\tilde{\Psi}_{N}^{(1)}\right\rangle$. Note that the first-order wave functions are generally not orthogonal to each other, and the shift correction in the effective Hamiltonian is therefore only invariant if the full overlap matrix $\left\langle\tilde{\Psi}_{M}^{(1)} \mid \tilde{\Psi}_{N}^{(1)}\right\rangle$ is included in the last term of $\left(H_{\text {eff }}\right)_{M N}$. Previously, ${ }^{26}$ we used only the diagonal $(M=N)$ part of the correction term [the last term in eqn (8)], which was, in turn, the default choice for the standard MS-CASPT2 (which we hereafter denote 'standard shifts'). The fully invariant inclusion has turned out to be of vital importance as we will see numerically later (hereafter denoted as 'invariant shifts').

The modification of the analytic nuclear energy gradient code due to the change of the level shift formula has been trivial. The computer code that implements the above methods is available in the latest release of MOLPRo. ${ }^{52}$ Other choices of the zeroth-order Hamiltonian, such as the so-called IPEA shift, ${ }^{53}$ can also be used in conjunction with XMS-CASPT2. However, the standard zeroth-order Hamiltonian has been used throughout the current work.

\subsection{Simulation of $1^{1} B_{3 u}$ and $1^{1} B_{2 u}$ continuous wave absorption spectra}

In this work, the vibronic coupling dynamics is treated in a quasidiabatic electronic basis $\left(\left\{\Phi_{N}(\boldsymbol{r}, \boldsymbol{Q})\right\}_{N=1,2}\right)$ since this simplifies numerical calculations. Here $\boldsymbol{r}$ denotes collectively the electronic coordinates and $\boldsymbol{Q}$ the set of vibrational normal coordinates. $\Phi_{1}$ and $\Phi_{2}$ correspond to the $1^{1} \mathrm{~B}_{3 \mathrm{u}}$ and $1^{1} \mathrm{~B}_{2 \mathrm{u}}$ excited states of pyrazine, respectively. The definition and generation of these quasi-diabatic states from the adiabatic wavefunctions $\Psi_{N}$ is described in ref. 48. Expanding $\left\langle\Phi_{N}(\boldsymbol{Q}) \mid\left(\hat{H}(\boldsymbol{Q}) \mid \Phi_{M}(\boldsymbol{Q})\right)\right\rangle-V_{0}(\boldsymbol{Q}) \delta_{N M}$ ( $\hat{H}$ denotes the electronic Hamiltonian) around the $\mathrm{S}_{0}$ equilibrium geometry $[\boldsymbol{Q}=0]$ to second order (except for bilinear terms) leads to the approximate two-state multimode matrix Hamiltonian of vibronic coupling theory (Table 1), ${ }^{54}$ in which $\gamma_{t}^{(n)}$ and $\gamma_{10 a}^{(n)}$ are parameters corresponding to the diagonal elements of the matrix of second derivatives of the excitation energies of the $1^{1} B_{3 u}$ and $1^{1} \mathrm{~B}_{2 \mathrm{u}}$ states with respect to the normal coordinates $Q_{t}$ and $Q_{10 a}$, respectively. $\hat{T}_{\text {nuc }}$ is the nuclear kinetic energy operator, and $V_{0}(\boldsymbol{Q})$ is the nuclear ground-state potential of pyrazine in the harmonic approximation. $Q_{i}$ denotes a dimensionless normal coordinate. $E_{n}$ are the vertical excitation energies, $\kappa_{t}^{(n)}$ the first-order intrastate and $\lambda_{10 a}$ the first-order $1^{1} \mathrm{~B}_{3 \mathrm{u}}-1^{1} \mathrm{~B}_{2 \mathrm{u}}$ interstate electron-vibrational coupling constants. The summation index $t$ includes all five totally symmetric or "tuning" coordinates of pyrazine: $Q_{1}, Q_{2}, Q_{6 a}$, $Q_{8 a}$ and $Q_{9 a}$. Tuning modes $\nu_{t}$ are characterized by modulating the energy gap between the two states. ${ }^{54}$ For symmetry reasons, only nuclear displacements that transform according to the $\mathrm{A}_{\mathrm{g}}$ and $\mathrm{B}_{1 \mathrm{~g}}$ irreducible representations of the $D_{2 \mathrm{~h}}$ point group can couple linearly to one-photon transitions into the $1^{1} B_{3 u}$ and $1^{1} B_{2 u}$ states.
The parameters $\left(\kappa_{t}^{(n)}, \lambda_{10 a}, \gamma_{t}^{(n)}\right.$ and $\left.\gamma_{10 a}^{(n)}\right)$ are computed by the same procedure as in ref. 48 , see also below.

The photoabsorption line spectrum is computed using Fermi's golden rule, ${ }^{55}$

$$
I(\omega) \propto \sum_{f}\left|\left\langle\Psi_{0}\left|\hat{\boldsymbol{H}}_{\mathrm{int}}\right| \Psi_{f}\right\rangle\right|^{2} \delta\left(\omega-E_{f}+E_{0}\right)
$$

where $\left|\Psi_{0}\right\rangle$ is the vibronic ground state of pyrazine with energy $E_{0}$, and $\left|\Psi_{f}\right\rangle$ are the final vibronic states of pyrazine with energies $E_{f}$. The operator $\hat{\boldsymbol{H}}_{\text {int }}$ describes the interaction of the molecule with the external electric field $\boldsymbol{E}(t)$ :

$$
\hat{\boldsymbol{H}}_{\text {int }}=-\sum_{N=1,2}\left|\Phi_{N}\right\rangle \boldsymbol{\mu}_{N 0} \boldsymbol{E}(t)\left\langle\Phi_{0}\right|+\text { h.c. },
$$

where $\boldsymbol{\mu}_{N o}$ are the nonvanishing transition moments in the diabatic representation.

The eigenvalues of the approximate two-state matrix Hamilton operator $\hat{\boldsymbol{H}}_{\mathrm{vib}}$ [Table 1] are obtained by numerically solving the time-independent vibronic Schrödinger equation with a vibronic basis, ${ }^{56}$

$$
\left|\Psi_{f}\right\rangle=\sum_{N=1,2} \sum_{v} C_{N v}^{f}\left|\Phi_{N}\right\rangle|v\rangle
$$

which is a complete direct-product basis of diabatic electronic states $\left|\Phi_{N}\right\rangle$ and eigenfunctions $\left|v_{i}\right\rangle$ of one-dimensional harmonic oscillators. Note that the matrix representation of $\hat{\boldsymbol{H}}_{\mathrm{vib}}$ decouples into two submatrices $\boldsymbol{H}_{1}$ and $\boldsymbol{H}_{2}$, corresponding to the vibronic levels of $\mathrm{B}_{3 \mathrm{u}}$ and $\mathrm{B}_{2 \mathrm{u}}$ symmetry, respectively.

The ground state $\left|\Psi_{0}\right\rangle$ of pyrazine is to a good approximation a Born-Oppenheimer product:

$$
\left|\Psi_{0}\right\rangle=\left|\Phi_{0}\right\rangle|\mathbf{0}\rangle
$$

where $\left|\Phi_{0}\right\rangle$ and $|\mathbf{0}\rangle$ denote the electronic and the vibrational ground states, respectively. The final vibronic states $\left|\Psi_{f}\right\rangle$, on the other hand, are given by eqn (11). $\hat{\boldsymbol{H}}_{\text {int }}$ is treated in the dipole approximation. Applying the Condon approximation in the diabatic basis, the transition matrix element from eqn (9) becomes $^{54}$

$$
\left\langle\Psi_{0}\left|\hat{\boldsymbol{H}}_{\mathrm{int}}\right| \Psi_{f}\right\rangle \propto \tau_{1} C_{1 \mathbf{0}}^{f}+\tau_{2} C_{2 \mathbf{0}}^{f},
$$

where only the first component $C_{N 0}^{f}$ of the eigenvectors of the submatrices $\boldsymbol{H}_{n}(n=1,2)$ determines the intensity of the $f$ th line. Obviously, this drastically reduces the numerical effort. In all computations of the present study, the oscillator strengths $\tau_{1}=0.1$ and $\tau_{2}=1.0$ are assumed for the vibronic transitions. The ratio of these values corresponds to the experimental observation. In ref. 47 , a ratio $\tau_{1}: \tau_{2}$ of $1: 4.7$ has been estimated by employing the same basis set (basis version 1) and active space definition as for the calculation of vibronic parameters in the present study. The oscillator strengths were

Table 1 The approximate two-state Hamiltonian of vibronic coupling theory. See the main text for the definition of the parameters

$$
\hat{\boldsymbol{H}}_{\mathrm{vib}}=\left(\hat{T}_{\mathrm{nuc}}+V_{0}(\boldsymbol{Q})\right) \mathbf{1}_{2}+\left[\begin{array}{cc}
E_{1}+\sum_{t}\left[\kappa_{t}^{(1)} Q_{t}+\gamma_{t}^{(1)} Q_{t}^{2}\right]+\gamma_{10 a}^{(1)} Q_{10 a}^{2} & \lambda_{10 a} Q_{10 a} \\
\lambda_{10 a} Q_{10 a} & E_{2}+\sum_{t}\left[\kappa_{t}^{(2)} Q_{t}+\gamma_{t}^{(2)} Q_{t}^{2}\right]+\gamma_{10 a}^{(2)} Q_{10 a}^{2}
\end{array}\right] .
$$


computed by combining transition dipole moments and vertical excitation energies obtained at the CASSCF and CASPT2 levels of theory, respectively. ${ }^{47}$

\section{Numerical results}

Our numerical demonstration in this section is threefold. In Section 3.1 we will first show that the artifacts one encounters with the standard MS-CASPT2 method in regions that are characterized by very small energy differences between two or more CASSCF reference states can be removed by using the new XMS-CASPT2 method. Secondly, in Section 3.2 we demonstrate the importance of the invariant inclusion of level shifts when the Fock operator has degenerate eigenvalues near state crossings. Finally, based on the two-state vibronic model, we simulate the CW photoabsorption spectra of the $1^{1} B_{3 u}$ and $1^{1} B_{2 u}$ states of pyrazine and compare the results to experiment as well as to previous theoretical spectra ${ }^{48,49}$ (Section 3.3).

We have used Dunning's augmented triple- $\zeta$ basis set (augcc-pVTZ), without the diffuse functions of highest angular shells for each atom. In addition, we included four $s$-, three $p$-, and three $d$-type diffuse functions located at the center of mass of the molecule, whose exponents are $s(0.021,0.008,0.0025$, $0.0008), p(0.017,0.009,0.004), d(0.015,0.008,0.004)$. This basis set has been employed in the calculations reported in ref. 47 (basis version 1). In principle, the aug-cc-pVTZ basis set would be sufficient for the description of the $1^{1} \mathrm{~A}_{\mathrm{g}}, 1^{1} \mathrm{~B}_{3 \mathrm{u}}$ and $1^{1} \mathrm{~B}_{2 \mathrm{u}}$ states of pyrazine, but we prefer this basis for consistency reasons.

The ground state geometry and force field have been determined by the single-state CASPT2 approach. ${ }^{57}$ The $1^{1} \mathrm{~B}_{3 \mathrm{u}}$ and $1^{1} \mathrm{~B}_{2 \mathrm{u}}$ excited states transform according to the $\mathrm{B}_{\mathrm{u}}$ irreducible representation if the $C_{2 \mathrm{~h}}$ point group applies to the nuclear frame. They are computed with state averaging and multi-state treatments within the reduced symmetry. The active space was constructed from 10 electrons in 8 orbitals ( 2 orbitals each in $a_{g}, a_{u}, b_{u}$, and $b_{g}$ within $C_{2 \mathrm{~h}}$ symmetry). ${ }^{47-49}$ A level shift of 0.3 $E_{\mathrm{h}}$ was used to avoid intruder state problems.

Note that although the $1^{1} \mathrm{~B}_{3 \mathrm{u}}$ and $1^{1} \mathrm{~B}_{2 \mathrm{u}}$ excitations are frequently assigned as the $S_{1}$ and $S_{2}$ states at the ground state equilibrium geometry and the $1^{1} B_{3 u}$ state is indeed the $S_{1}$ state, the true second excited state is of $A_{u}$ symmetry and $1^{1} B_{2 u}$ is in reality $\mathrm{S}_{3} \cdot{ }^{47}$ We therefore use $1^{1} \mathrm{~B}_{3 \mathrm{u}}$ and $1^{1} \mathrm{~B}_{2 \mathrm{u}}$ to label these states in this article.

\subsection{Excited state potential energy surfaces in the Franck-Condon region}

First we present the PESs of the $1^{1} B_{3 u}$ and $1^{1} B_{2 u}$ states of pyrazine near the ground-state equilibrium geometry. Fig. 1 compares those computed by the standard MS-CASPT2 and those by XMS-CASPT2. Apparently, the PESs from MS-CASPT2 have artifacts around $Q_{10 a}=0$ and $Q_{6 a}=-0.5$, whereas those from XMS-CASPT2 do not exhibit such erratic behavior. The artifacts are ascribed to the rotation of the reference functions due to a state crossing of the reference surfaces, as in the examples reported earlier. ${ }^{25,26}$ This is supported by Fig. 2, which shows the two-dimensional cut of the reference PESs along the $Q_{6 a}$ and $Q_{10 a}$ modes.

In Fig. 3 we present a one-dimensional cut of the same PESs along the $Q_{10 a}$ coordinate computed by MS-CASPT2 and XMSCASPT2, as well as by MRCI+Q. ${ }^{58,59}$ The curves computed by the standard MS-CASPT2 have an artificial dent near the equilibrium structure, which is not present in the MRCI+Q curves. The XMS-CASPT2 method removes this artifact and the curves are almost parallel to the one from MRCI+Q. At the equilibrium geometry, the MS-CASPT2 and XMS-CASPT2 energies become identical since the off-diagonal element of the Fock operator in the reference space (i.e., $\langle M|\hat{\mid}| N\rangle$ with $M \neq N$ ) is zero due to symmetry. Therefore, the difference in the separation of two excited states is ascribed to the insufficient treatment of dynamical correlation at the CASPT2 level (and not to the multi-state treatment). The quality of XMS-CASPT2 PESs will be tested in what follows.

\subsection{Conical intersection}

Fig. 4 shows a one-dimensional cut of the same PESs along the $Q_{10 a}$ mode near the conical intersection $\left[Q_{6 a}=-3.9\right]$. The lower panel corresponds to the zeroth-order energies (i.e., the eigenvalues of the Fock matrix in the reference space), showing that it has degenerate eigenvalues near the state crossing. The standard MS-CASPT2 has a smooth potential here (red curves). The erratic behavior of the XMS-CASPT2 surfaces with the standard level shift (black curves) is attributed to the degeneracy of the eigenvalues of the Fock matrix: at the $Q_{10 a}=0$ geometry, the rotation angle in XMS is zero due to symmetry; but when the geometry is slightly distorted along the $Q_{10 a}$ mode, the offdiagonal Fock elements become nonzero, while the difference between two diagonal elements remains small. This leads to significant rotation in XMS, and hence the artifact as shown in the upper panel of Fig. 4. This problem has been fixed by using the invariant level shift (blue curves). Note that the curves from XMS-CASPT2 with the standard and invariant level shifts become nearly identical apart from the crossing region. These observations are confirmed by the two-dimensional cuts in Fig. 5.

\subsection{Simulation of $1^{1} B_{3 u}$ and $1^{1} B_{2 u}$ continuous wave photoabsorption spectra}

The parameters $\kappa_{t}^{(n)}$ and $\gamma_{t}^{(n)}$ for the $1^{1} \mathrm{~B}_{3 \mathrm{u}}$ and $1^{1} \mathrm{~B}_{2 \mathrm{u}}$ states evaluated with the single-state CASPT2 method are compiled in the ESI. $\dagger^{60}$ Since the $1^{1} \mathrm{~B}_{3 \mathrm{u}}$ and $1^{1} \mathrm{~B}_{2 \mathrm{u}}$ states cannot interact within $D_{2 \mathrm{~h}}$ symmetry, a state-averaged treatment of both states is not necessary in this case. We have verified that the CASPT2 excitation energies of both states computed in the $D_{2 \mathrm{~h}}$ point group are very insensitive to the optimization scheme applied to the reference functions: individually converged CASSCF wave functions yield single-state CASPT2 excitation energy functions that are almost identical to those obtained by MS-CASPT2 calculations based on reference functions that are obtained by including the $1^{1} \mathrm{~B}_{3 \mathrm{u}}$ and $1^{1} \mathrm{~B}_{2 \mathrm{u}}$ states with equal weights in the CASSCF energy functional.

Since the MS-CASPT2 approach yields artifacts in the adiabatic PESs of the $1^{1} \mathrm{~B}_{\mathrm{u}}$ and $2{ }^{1} \mathrm{~B}_{\mathrm{u}}$ states for nonzero displacements along $Q_{10 a}$ in the vicinity of the degeneracy of the CASSCF 

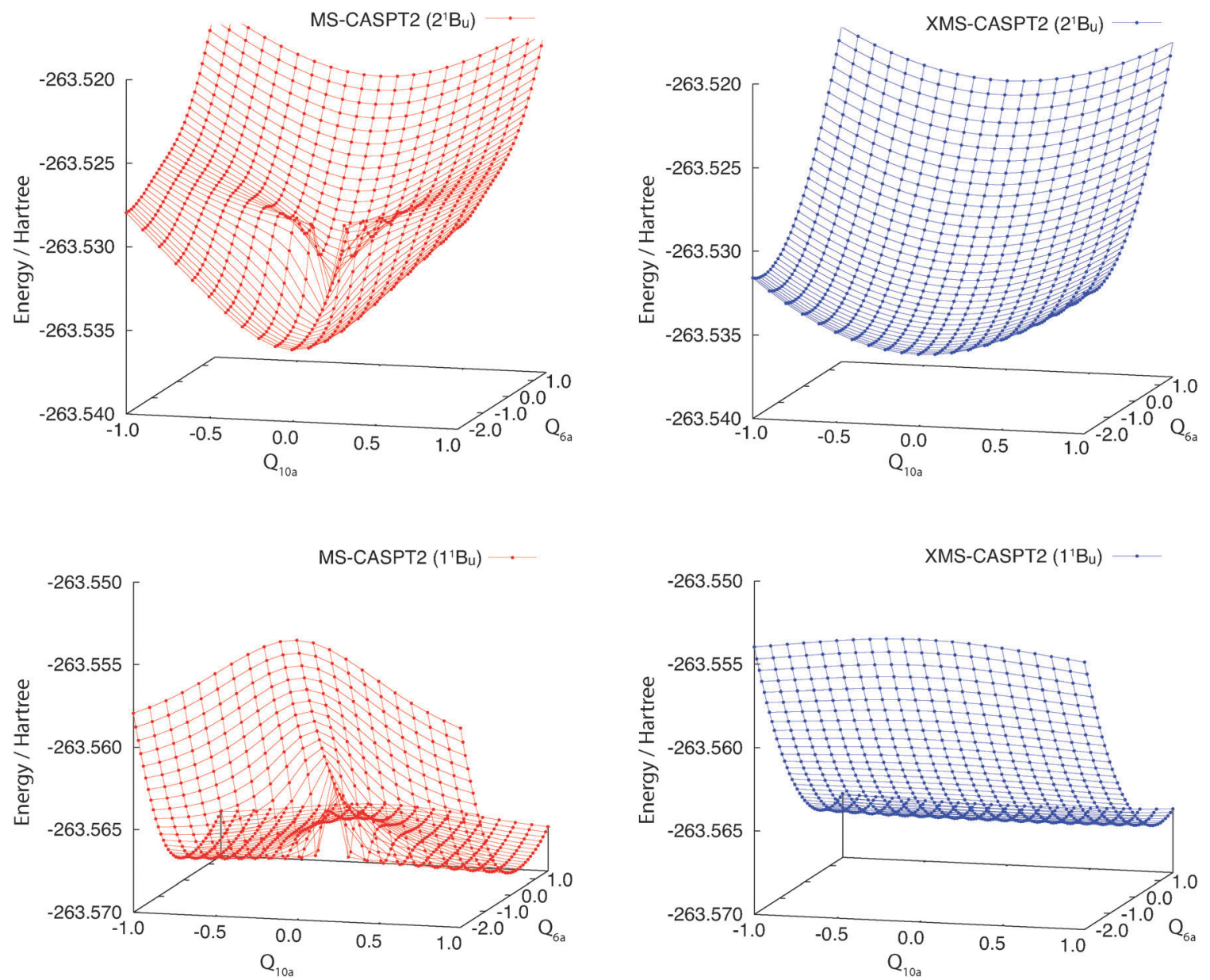

Fig. 1 Potential energy surfaces of the adiabatic $1^{1} B_{u}$ and $2^{1} B_{u}$ states of pyrazine (using $C_{2 h}$ labels) are shown in the Franck-Condon region. The electronic states are computed at the standard (left) and extended (right) MS-CASPT2 levels of theory. The nuclear coordinate space is spanned by $Q_{6 a}$ and $Q_{10 a}$ Note that at $Q_{10 a}=0$, MS- and XMS-CASPT2 give the same results due to symmetry.

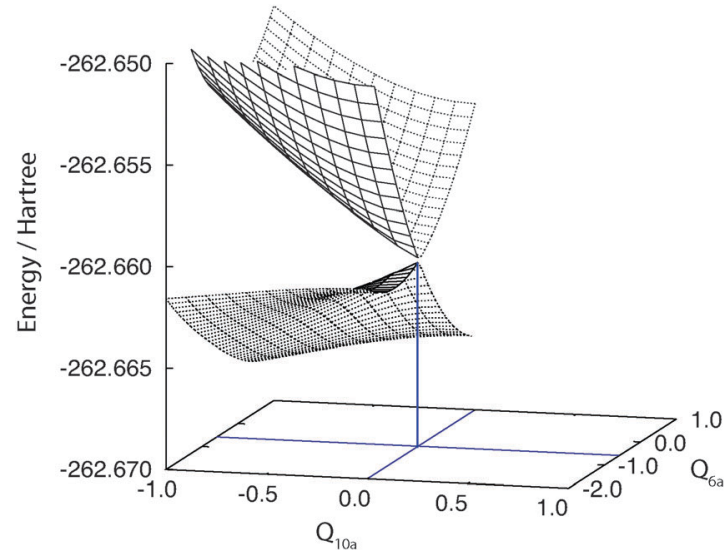

Fig. 2 The state crossing of the SA-CASSCF potential energy surfaces, which coincides with the artifacts in the standard MS-CASPT2. The surfaces are symmetric with respect to the inversion of the $Q_{10 a}$ axis.

reference states as we demonstrated above, a reliable determination of the $Q_{10 a}$ vibronic coupling parameters is not possible. We therefore concentrate only on a comparison of spectra calculated using MRCI and XMS-CASPT2 parameters.

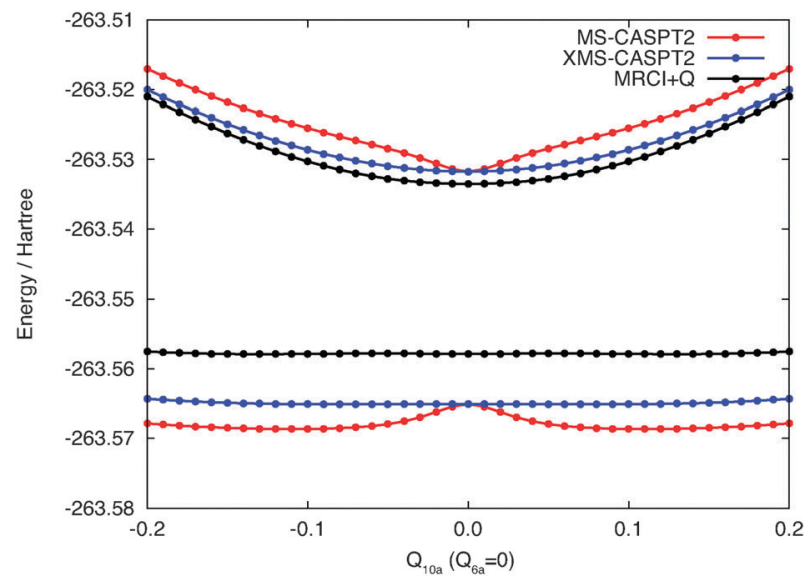

Fig. 3 Potential energy curves along the $Q_{10 a}$ mode computed by MS-CASPT2, XMS-CASPT2 and MRCI+Q.

The $\kappa_{t}^{(n)}$ parameters computed by XMS-CASPT2 show qualitative agreement with those by MRCI: ${ }^{49}$ for instance, $\kappa_{6 a}^{(1)} / \kappa_{6 a}^{(2)}$ and $\kappa_{8 a}^{(1)} / \kappa_{8 a}^{(2)}$ have similar values. However, the magnitude of the 

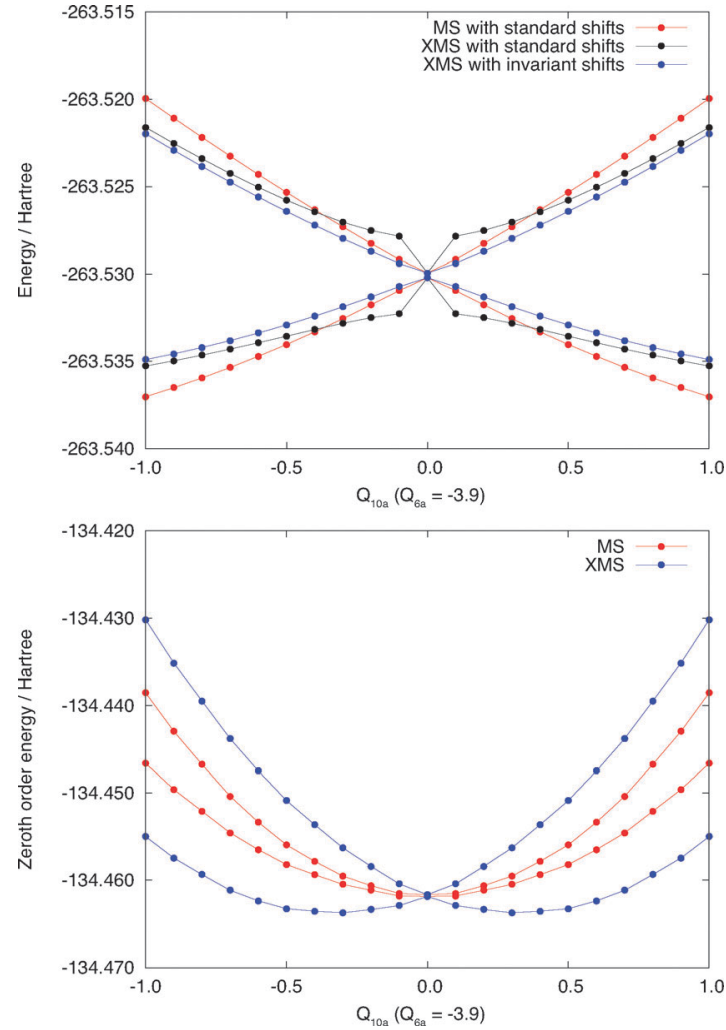

Fig. 4 Upper panel: a one dimensional cut of the potential energy surfaces along the $Q_{10 a}$ mode near the conical intersection $\left(Q_{6 a}=-3.9\right)$, computed by MS-CASPT2 and XMS-CASPT2 with the standard and invariant level shift of $0.3 E_{\mathrm{h}}$. Lower panel: the zeroth-order energies along the same cut.

individual parameters differs significantly in some cases; the most pronounced example is $\kappa_{1}^{(1)}$ (MRCI: $-0.0470 \mathrm{eV}$, CASPT2: $-0.0299 \mathrm{eV})$. The set of vibronic coupling parameters for $\hat{\boldsymbol{H}}_{\text {vib }}$ (Table 1) is completed by $\lambda_{10 a}=0.201 \mathrm{eV}, \gamma_{10 a}^{(1)}=-0.014 \mathrm{eV}$ and $\gamma_{10 a}^{(2)}=-0.014 \mathrm{eV} . \lambda_{10 a}, \gamma_{10 a}^{(1)}$, and $\gamma_{10 a}^{(2)}$ have been determined by a least-squares fit of the eigenvalues of the two-state model potential energy matrix as a function of $Q_{10 a}$ at the XMS-CASPT2 level of theory. The XMS-CASPT2 parameters deviate only slightly

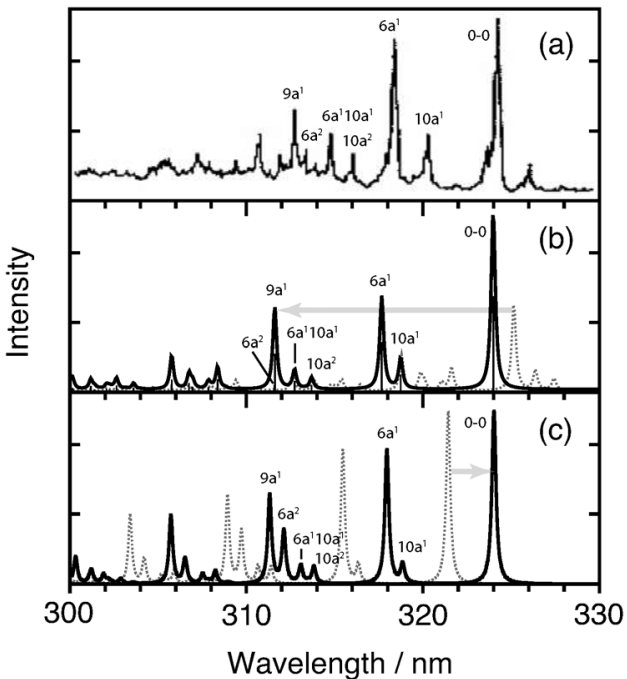

Fig. $6 \mathrm{CW}$ photoabsorption spectra of the $1^{1} \mathrm{~B}_{3 \mathrm{u}}$ state: (a) experimental, ${ }^{61}$ (b) XMS-CASPT2, and (c) MRCI (based on ref. 49). Unshifted (dotted) and shifted (full) theoretical spectra are shown. See text for details. The assignments in the experimental spectrum are based on an analysis of both theoretical spectra, taking available information on experimental $1^{1} B_{3 u}$ frequencies into account.

from those obtained with the MRCI method $(0.1825 \mathrm{eV},-0.0180 \mathrm{eV}$, $-0.0180 \mathrm{eV}$, respectively). ${ }^{48}$

Fig. 6 and 7 show the observed, XMS-CASPT2, and MRCI photoabsorption spectra of the $1^{1} \mathrm{~B}_{3 \mathrm{u}}$ and $1^{1} \mathrm{~B}_{2 \mathrm{u}}$ states, respectively. The coherence-decay constants are set to $T_{2}=400 \mathrm{fs}$ and $T_{2}=35$ fs, respectively, to account for homogeneous broadening of the individual vibronic transitions. For a discussion of the microscopic origin of dephasing rates $1 / T_{2}$ see, for instance, ref. 62. The theoretical spectra shown as solid lines in panel (b) of Fig. 6 and 7 have been blue-shifted by $0.165 \mathrm{eV}$ and by $0.096 \mathrm{eV}$, respectively, in order to compensate for the underestimation of 0-0 transitions of both bands by the CASPT2 method. The MRCI-derived spectrum (solid line) is likewise red-shifted by $0.031 \mathrm{eV}$ in Fig. 6(c). It is typical that CASPT2 underestimates excitation energies, while MRCI slightly overestimates them.
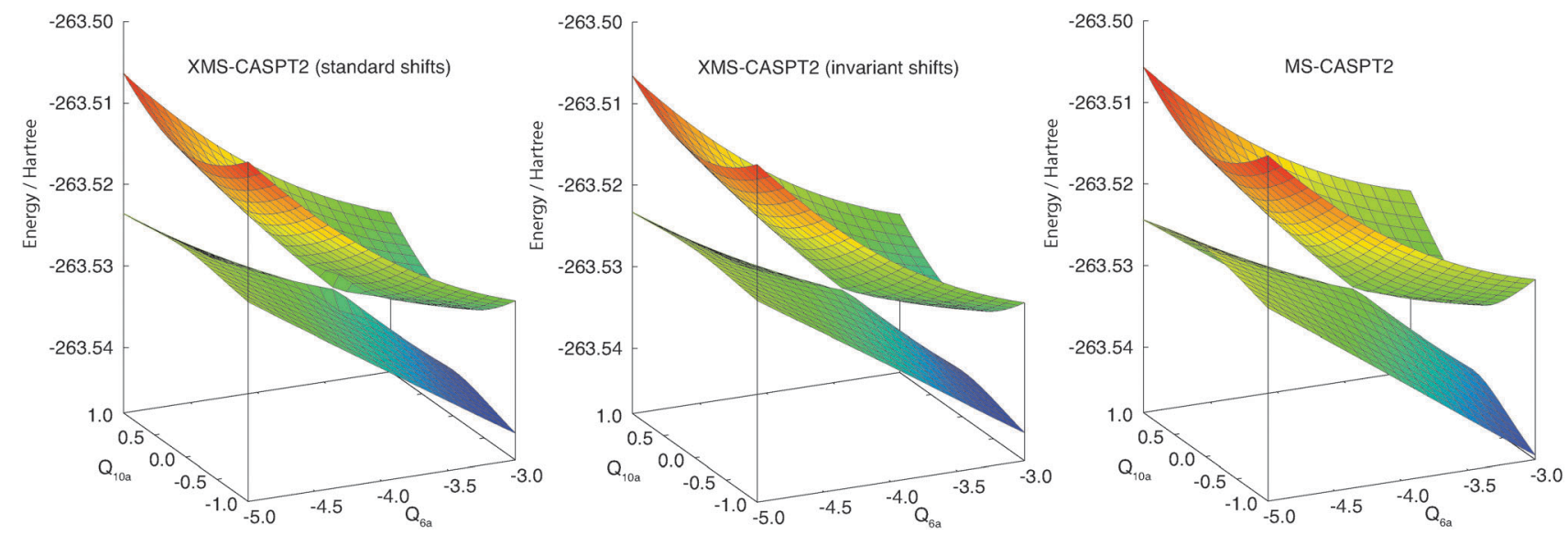

Fig. 5 Two-dimensional maps with $Q_{6 a}$ and $Q_{10 a}$ around the conical intersection computed by MS-CASPT2 and XMS-CASPT2. Note that MS-CASPT2 and XMS-CASPT2 are identical when $Q_{10 a}=0$ due to symmetry. 


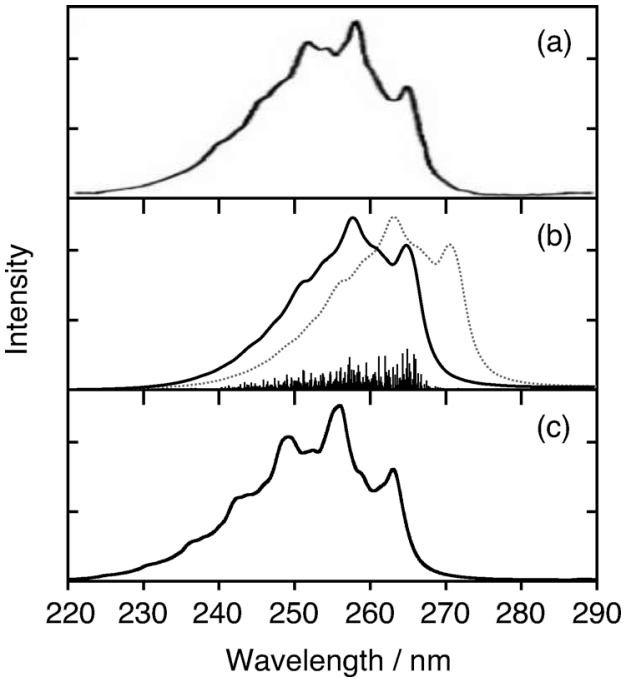

Fig. $7 \mathrm{CW}$ photoabsorption spectra of the $1^{1} \mathrm{~B}_{2 \mathrm{u}}$ state: (a) experimental, ${ }^{31}$ (b) XMS-CASPT2, and (c) MRCI (based on ref. 49). Unshifted (dotted) and shifted (full) theoretical spectra are shown. See text for details.

Note that the XMS-CASPT2 spectra in this work are computed with a vibronic model that slightly differs from the vibronic Hamiltonians used in the previous MRCI simulations. ${ }^{48,49}$ In ref. 48 a 4-mode model was used, which included the $Q_{1}, Q_{6 a}, Q_{9 a}$ tuning modes, as well as the $Q_{10 a}$ coupling mode. In ref. 49 the 4-mode model was augmented by including also the $Q_{8 a}, Q_{4}$, and $Q_{14}$ modes, but the $Q_{2}$ tuning mode was still neglected. In the current work we employ a 6-mode model that includes all five $\mathrm{A}_{\mathrm{g}}$ tuning modes $\left(Q_{1}, Q_{2}, Q_{6 a}, Q_{8 a}, Q_{9 a}\right)$, but neglects the quadratic effect of the out-of-plane displacements $Q_{4}$ and $Q_{14}$. Secondly, in this study the ground-state force field is consistently constructed by CASPT2, while a second-order Møller-Plesset (MP2) ground-state force field was used in ref. 49. Third, diagonal quadratic intrastate coupling terms with parameters $\gamma_{t}^{(n)}$ have been introduced for the $\mathrm{A}_{\mathrm{g}}$ vibrations, which were absent in the model of ref. 49 .

The $1^{1} \mathrm{~B}_{3 \mathrm{u}}$ and $1{ }^{1} \mathrm{~B}_{2 \mathrm{u}}$ MRCI absorption spectra shown in Fig. 6(c) and $7(\mathrm{c})$, respectively, have been calculated with the MRCI parameters and the 7-mode vibronic model reported in ref. 49. The $1^{1} \mathrm{~B}_{3 \mathrm{u}}$ spectrum was not shown in ref. 49 and is published for the first time in this work.

Both XMS-CASPT2 and MRCI successfully reproduce the main vibronic features of the $1^{1} B_{3 u}$ band (Fig. 6). The XMSCASPT2 spectrum appears to better describe the spacing between levels $\nu_{10 a}^{1}$ and $\nu_{6 a}^{1}$ and relative intensities of three pronounced excitations between $312 \mathrm{~nm}$ and $317 \mathrm{~nm}$. These three resonances in the experimental spectrum have originally been attributed to vibronic levels $\nu_{10 a}^{2}, \nu_{6 a}^{1} \nu_{10 a}^{1}$ and $\nu_{6 a}^{2}{ }^{31}$ An analysis of the strong transition at $311.59 \mathrm{~nm}$ in the XMSCASPT2 spectrum reveals, however, that the intensity is derived from two nearly degenerate levels of $B_{3 u}$ vibronic symmetry with an intensity ratio of $6.1\left(\nu_{9 a}^{1}\right)$ to $1.0\left(\nu_{6 a}^{2}\right)$. The present model therefore confirms the assignments of ref. 46 and 48 that the strong peak at around $312.5 \mathrm{~nm}$ in the experimental spectrum corresponds primarily to the quantum $\nu_{9 a}^{1}$.
This suggests the following interpretation of Fig. 6(b) and (c): the XMS-CASPT2 calculation wrongly attributes a slightly higher energy to the overtone $\nu_{6 a}^{2}$ as compared to the fundamental of $\nu_{9 a}$, and the small spacing between both transitions leads to an overestimation of the peak appearing at $c a$. $311.5 \mathrm{~nm}$ in Fig. 6(b). On the other hand, the MRCI model correctly predicts that the $\nu_{6 a}^{2}$ transition is red-shifted with respect to $\nu_{9 a}^{1}$ but strongly overestimates the intensity of $\nu_{6 a}^{2}$. We emphasize again that in the MRCI simulation of ref. 49 an MP2 ground state potential and a slightly different vibronic model than in the current work were used. For a more detailed and consistent analysis one would need to compute all three MRCI and XMS-CASPT2 potentials on equal footing and carry out the simulations using the same vibronic model. This is beyond the scope of the present paper and will be the subject of future work.

The XMS-CASPT2 simulation of the $1^{1} \mathrm{~B}_{2 \mathrm{u}}$ band is less satisfactory than the MRCI simulation (Fig. 7), although both electronic structure theories yield similar $Q_{10}$-related coefficients $\left(\lambda_{10 a}, \gamma_{10 a}^{(1)}\right.$ and $\left.\gamma_{10 a}^{(2)}\right)$. The vibronic models yield the following intersection points: $Q_{1}=6.8, Q_{6 a}=-3.7$ (XMSCASPT2) and $Q_{1}=5.4, Q_{6 a}=-3.9$ (MRCI). This result implicates that the discrepancy between XMS-CASPT2- and MRCI-based spectra is partly owing to a substantial difference between the CASPT2- and MRCI-derived topologies of the $1^{1} \mathrm{~B}_{3 \mathrm{u}}-1^{1} \mathrm{~B}_{2 \mathrm{u}}$ degeneracy subspaces with respect to the location of the ground-state equilibrium geometry.

\section{Conclusions}

In this paper we have demonstrated that the recently developed XMS-CASPT2 method is a useful tool for the ab initio description of strongly coupled electronically excited states, even in cases where the standard MS-CASPT2 approach completely fails. For the pyrazine excited states that are considered here, the standard MS-CASPT2 gives a hump around the equilibrium geometry of the ground state that is associated with a CASSCF state crossing, while XMS-CASPT2 is free from such artifacts. We have also shown the importance of including the level shift in a way that is invariant with respect to unitary transformations of the reference functions.

The smooth PES computed by XMS-CASPT2 has allowed us to examine the accuracy of the vibronic interaction strength of the $1^{1} \mathrm{~B}_{3 \mathrm{u}}$ and $1^{1} \mathrm{~B}_{2 \mathrm{u}}$ states of pyrazine, which was not possible with the standard MS-CASPT2 method. We have extended previous versions ${ }^{46,48,49}$ of the model Hamiltonian for nonadiabatic dynamics in the coupled $1^{1} \mathrm{~B}_{3 \mathrm{u}}$ and $1^{1} \mathrm{~B}_{2 \mathrm{u}}$ vibronic manifolds by including the diagonal quadratic intrastate coupling parameters $\gamma_{t}^{(n)}$ for the $\mathrm{A}_{\mathrm{g}}$ modes.

A comparison of the simulated $1^{1} \mathrm{~B}_{3 \mathrm{u}}$ absorption bands by XMS-CASPT2 and by MRCI ${ }^{49}$ shows that while some aspects of the vibronic structure are improved in the XMS-CASPT2 spectrum, other features of the experimental envelope are better approximated by the MRCI-based approach. A more detailed analysis of the influence of the PESs and of the different vibronic models on the simulated spectra would require 
computation of an accurate MRCI PES for the electronic ground state, as well as extension of the existing MRCI potentials by the $Q_{2}$ mode that was neglected in previous work. ${ }^{49}$ This will be the subject of future work.

We have also observed that the XMS-CASPT2 $1^{1} \mathrm{~B}_{2 \mathrm{u}}$ spectrum is slightly inferior to that computed with MRCI parameters, which can probably be ascribed to a lower accuracy of the XMSCASPT2 potential energy surfaces. Nevertheless, we conclude that the XMS-CASPT2 method is a useful method for such simulations when the system of concern becomes so large that more accurate MRCI computations are too expensive.

\section{Acknowledgements}

This work has been supported by the Deutsche Forschungsgemeinschaft as part of the SimTech Cluster of Excellence at the University of Stuttgart. T.S. thanks the Japan Society for the Promotion of Science for financial support. C.W. has received continued support from the Mohn Foundation (Grant No. A32542) and the Centre for Theoretical and Computational Chemistry (CTCC) at the University of Tromsø. We acknowledge computational resources provided by Norwegian High Performance Computing (NOTUR).

\section{References}

1 B. O. Roos, P. Linse, P. E. M. Siegbahn and M. R. A. Blomberg, Chem. Phys., 1982, 66, 197-207.

2 K. Andersson, P.-Å. Malmqvist, B. O. Roos, A. J. Sadlej and K. Wolinski, J. Phys. Chem., 1990, 94, 5483-5488.

3 K. Andersson, P.A. Malmqvist and B. O. Roos, J. Chem. Phys., 1992, 96, 1218-1226.

4 H.-J. Werner, Mol. Phys., 1996, 89, 645-661.

5 J. Finley, P.-Å. Malmqvist, B. O. Roos and L. Serrano-Andrés, Chem. Phys. Lett., 1998, 288, 299-306.

6 P. Celani and H.-J. Werner, J. Chem. Phys., 2000, 112, 5546-5557.

7 C. Angeli, R. Cimiraglia, S. Evangelisti, T. Leininger and J.-P. Malrieu, J. Chem. Phys., 2001, 114, 10252-10264.

8 F. Aquilante, P.-A. Malmqvist, T. B. Pedersen, A. Ghosh and B. O. Roos, J. Chem. Theory Comput., 2008, 4, 694-702.

9 T. Shiozaki and H.-J. Werner, J. Chem. Phys., 2010, 133, 141103. 10 W. Meyer, Methods of Electronic Structure Theory, Plenum, New York, 1977. 11 H.-J. Werner and E.-A. Reinsch, J. Chem. Phys., 1982, 76, 3144-3156. 12 K. Hirao, Chem. Phys. Lett., 1992, 190, 374-380.

13 K. Hirao, Chem. Phys. Lett., 1992, 196, 397-403.

14 K. Hirao, Chem. Phys. Lett., 1993, 201, 59-66.

15 H. Nakano, J. Chem. Phys., 1993, 99, 7983-7992.

16 S. Ten-no, Chem. Phys. Lett., 2007, 447, 175-179.

17 Y. Kurashige and T. Yanai, J. Chem. Phys., 2011, 135, 094104.

18 D. Zgid and M. Nooijen, J. Chem. Phys., 2008, 128, 144116.

19 D. Ghosh, J. Hachmann, T. Yanai and G. K.-L. Chan, J. Chem. Phys., 2008, 128, 144117.

20 P. Pulay, Int. J. Quantum Chem., 2011, 111, 3273-3279.

21 B. Huron, J. P. Malrieu and P. Rancure, J. Chem. Phys., 1973, 58, 5745-5759.

22 Y. G. Khait, J. Song and M. R. Hoffmann, J. Chem. Phys., 2002, 117, 4133-4145.

23 T. J. Dudley, Y. G. Khait and M. R. Hoffmann, J. Chem. Phys., 2003, 119, 651-660.

24 L. Serrano-Andrés, M. Merchán and R. Lindh, J. Chem. Phys., 2005, 122, 104107.

25 A. A. Granovsky, J. Chem. Phys., 2011, 134, 214113.
26 T. Shiozaki, W. Győrffy, P. Celani and H.-J. Werner, J. Chem. Phys., 2011, 135, 081106.

27 P. Celani and H.-J. Werner, J. Chem. Phys., 2003, 119, 5044-5057.

28 K. K. Innes, I. G. Ross and W. R. Moomaw, J. Mol. Spectrosc., 1988, 132, 492-554.

29 A. Bolovinos, P. Tsekeris, J. Philips, E. Pantos and G. Anditsopouluos, J. Mol. Spectrosc., 1984, 103, 240-256.

30 J. Kommandeur, W. A. Majewski, W. L. Meerts and D. W. Pratt, Annu. Rev. Phys. Chem., 1987, 38, 433-462.

31 I. Yamazaki, T. Murao, T. Yamanaka and K. Yoshihara, Faraday Discuss. Chem. Soc., 1983, 75, 395-405.

32 M. H. Palmer and I. C. Walker, Chem. Phys., 1991, 157, 187-200.

33 P. Weber and J. R. Reimers, J. Phys. Chem. A, 1999, 103, 9821-9829.

34 Y. Li, J. Wan and X. Xu, J. Comput. Chem., 2007, 28, 1658-1667.

35 A. Raab, G. A. Worth, H.-D. Meyer and L. S. Cederbaum, J. Chem. Phys., 1999, 110, 936-946.

36 M. P. Fülscher, K. Andersson and B. O. Roos, J. Phys. Chem., 1992, 96, 9204-9212.

37 K. Andersson, Theor. Chim. Acta, 1995, 91, 31-46.

38 M. P. Fülscher and B. O. Roos, Theor. Chim. Acta, 1994, 87, 403-413.

39 J. E. DelBene, J. D. Watts and R. J. J. Bartlett, J. Chem. Phys., 1997, 106, 6051-6060.

40 G. A. Worth, H.-D. Meyer and L. S. Cederbaum, J. Chem. Phys., 1996, 105, 4412-4426.

41 M. Schreiber, M. R. Silva-Junior, S. P. A. Sauer and W. Thiel, J. Chem. Phys., 2008, 128, 134110.

42 M. R. Silva-Junior, M. Schreiber, S. P. A. Sauer and W. Thiel, J. Chem. Phys., 2010, 133, 174318.

43 M. R. Silva-Junior, M. Schreiber, S. P. A. Sauer and W. Thiel, J. Chem. Phys., 2008, 129, 104103.

44 S. P. A. Sauer, M. Schreiber, M. R. Silva-Junior and W. Thiel, J. Chem. Theory Comput., 2009, 5, 555-564.

45 N. Heider and S. F. Fischer, Chem. Phys., 1984, 88, 209-220.

46 L. Seidner, G. Stock, A. L. Sobolewski and W. Domcke, J. Chem. Phys., 1992, 96, 5298-5309.

47 C. Woywod, A. Papp, G. J. Halasz and A. Vibok, Theor. Chem. Acc., 2010, 125, 521-533.

48 C. Woywod, W. Domcke, A. L. Sobolewski and H.-J. Werner, J. Chem. Phys., 1994, 100, 1400-1413.

49 G. Stock, C. Woywod, W. Domcke, T. Swinney and B. S. Hudson, J. Chem. Phys., 1995, 103, 6851-6860.

50 B. O. Roos and K. Andersson, Chem. Phys. Lett., 1995, 245, 215-223.

51 P. J. Knowles and H.-J. Werner, Theor. Chim. Acta, 1992, 84, 95-103.

52 H.-J. Werner, P. J. Knowles, G. Knizia and F. R. Manby, M. Schütz, et al., , MOLPRO, version 2012.1, a package of ab initio programs, Cardiff, UK, 2012, see http://www.molpro.net.

53 G. Ghigo, B. O. Roos and P.-Å. Malmqvist, Chem. Phys. Lett., 2004, 396, 142-149.

54 H. Köppel, W. Domcke and L. S. Cederbaum, Adv. Chem. Phys., 1984, 57, 59-246.

55 A. Lami, C. Petrongolo and F. Santoro, Conical Intersections, World Scientific, Singapore, 2004, p. 705.

56 C. Cohen-Tannoudji, B. Diu and F. Laloe, Quantum mechanics, Wiley, New York, 1977, vol. 1.

57 K. Andersson, M. R. A. Blomberg, M. P. Fülscher, G. Karlström, R. Lindh, P.-Å. Malmqvist, P. Neogrády, J. Olsen, B. O. Roos, A. J. Sadlej, M. Schütz, L. Seijo, L. Serrano-Andrés, P. E. M. Siegbahn and P.-O. Widmark, MOLCAS Version 7.4, Lund University, Sweden, 2010.

58 H.-J. Werner and P. J. Knowles, J. Chem. Phys., 1988, 89, 5803-5814.

59 P. J. Knowles and H.-J. Werner, Chem. Phys. Lett., 1988, 145, 514-522.

60 See ESI $\uparrow$ for the computational details of photoabsorption spectra.

61 M. Perić, S. D. Peyerimhoff and R. J. Buenker, Z. Phys. D: At., Mol. Clusters, 1992, 24, 177-198.

62 W. Domcke and G. Stock, Adv. Chem. Phys., 1997, 100, 1-169. 